\title{
FIELD OBSERVATIONS OF SEICHE EVENTS IN A T-SHAPE BAY OF KAMI-KOSHIKI ISLAND, JAPAN
}

\author{
Toshiyuki ASANO$^{1}$, Toru YAMASHIRO ${ }^{1}$ and Taro KAKINUMA ${ }^{1}$
}

\begin{abstract}
Field observations on secondary oscillations (seiche) in Urauchi Bay of Kami-koshiki Island, Japan were conducted during October 7-21, 2008. The seiches prone bay has a unique configuration as T-shape, which may cause the inherent characteristics of the eigen oscillations. The analyzed data show that the oscillations of the bay have at least two distinct modes; mode- 1 oscillation of the period around 25 minutes with forming anti-nodes at both inward inlets and possessing a node at the bay mouth, mode-2 oscillation of the period around 12 minutes which oscillates between both inward inlets having the node at the branching point. Next, numerical simulations were conducted to reproduce the secondary oscillations in the bay by providing input sinusoidal waves with the eigen periods of oscillations to the calculating bay geometry. The numerical results are found to show basically consistent characteristics with the field observations.
\end{abstract}

keywords : Secondary oscillation, seiche, coastal disaster, air-ocean coupling process, harbor resonance

\section{INTRODUCTION}

During winter and early spring time, large oscillations of water level (seiches) with period of several minutes to an hour are regularly happened in harbors, bays and straits along the west coast of Kyushu area, Japan. The seiche events are locally called as “abiki” waves, which literally mean net-dragging waves in Japanese. The events hamper ship loading and maneuvering or cause flooding, and furthermore capsize and damage fishing boat.

The generation mechanism has not been fully understood, but most of the relevant studies have pointed out that an abrupt atmospheric pressure disturbance with an amplitude of several hecto-Pascals is strongly related to its origin. Hibiya and Kajiura, (1982) developed a resonantly air-sea coupling model between traveling air pressure disturbance and generated long period water waves. Meanwhile, de Jong and Battjes (2004) attributed to low frequency wind fluctuation associated with trailing convection cells behind a cold front where the cold air moves over the relatively warm sea surface.

The atmospheric disturbance propagating above the ocean surface generates significant long ocean waves by the resonant mechanism when the atmospheric disturbance speed becomes closer to the long wave speed of the ocean waves. The amplified ocean waves arriving at the entrance of a semi-closed coastal basin can induce hazardous seiche event due to harbor resonance. Vilibic et al.(2008) have proposed a numerical model based on the above mechanism to reproduce a destructive seiche event occurred in Menorca, Balearic Islands, Spain, 2006 and found that the model results reproduced the observed sea level heights quantitatively.

On 24th February, 2009, Urauchi Bay, located on west coast of Kami-koshiki Island, south-east coast of Kyushu, was attacked by a destructive seiche event. Incoming long waves generated by atmospheric disturbance were amplified to 3.1 meters' high at the maximum which was observed at the inward part of Kojima inlet. Historically, the highest seiche in Japan was recorded as $278 \mathrm{~cm}$, in Nagashaki city, 1979, but this seiche event surpassed that. More than 18 boats were capsized, eight houses were flooded and huge fish cage systems storing tunas were damaged.

The authors have paid attention to the seiche events in Urauchi bay for recent years. The seiche-prone bay has a T-shape configuration and behaves a unique oscillation due to the topography. This paper is devoted to demonstrate the field observation results on the seiche motion in Urauchi bay which was conducted in October, 2008 just four month before the destructive seiche event in February 2009.

The present work investigates the characteristics of the secondary oscillations in Urauchi bay by field observations. This study focuses on the unique T-shape configuration of the bay and discusses the hydrodynamic characteristics in light of the eigen oscillations of the bay. Furthermore, numerical analyses to reproduce the unique oscillations in the bay are conducted and comparisons with the observed results are made.

\footnotetext{
${ }^{1}$ Division of Ocean Civil Engineering, Graduate School of Science and Technology, Kagoshima University 1-21-40, Korimoto, Kagoshima, 890-0065, JAPAN
} 


\section{MEASUREMENTS}

\section{Deployment of Instruments and Data Acquisition}

Figure 1 illustrates the location of the Koshiki-jima Islands and Fig. 2 shows the measuring points in Urauchi Bay area. The sea surface elevation measurements were conducted at the entrance point of the bay (St.1), the branching point (St. 2), the inside points of each inlet (Kuwano-ura inlet; St.3 and Kojima inlet; St.4) and at the Kojima fishing port (St.5). Four ultra-sonic velocity meters were also set at St.1 $\sim$ St.4. Since the sea surface elevations were detected by measuring total pressure(water pressure plus atmospheric pressure), a barometer should be equipped at St.5 to compensate the fluctuating atmospheric pressure. The field measurements were operated during Oct. 7 Oct. 21, 2008.

The sea level and current vector records were continuously taken with a sampling rate of 1 min. All the current velocities measured at St.1 St.4 show that the principal directions direct to the axis directions of the topography there. Typical raw data of the velocity fluctuations at St. $1 \sim$ St. 4 and the sea surface elevation at St.5 are shown in Fig.3. Here, the northward velocity is taken positive for the records at St. 1 and St.3, and the eastward velocity is taken positive for the records at St.2 and St.4. It is found from the elevation records at St.5 that several minutes to several ten minutes fluctuations are superimposed on the semi-dirnal tidal fluctuations. The heights of secondary oscillations are found to reach around $50 \mathrm{~cm}$ at 10:53 and 14:09 on 14 October.

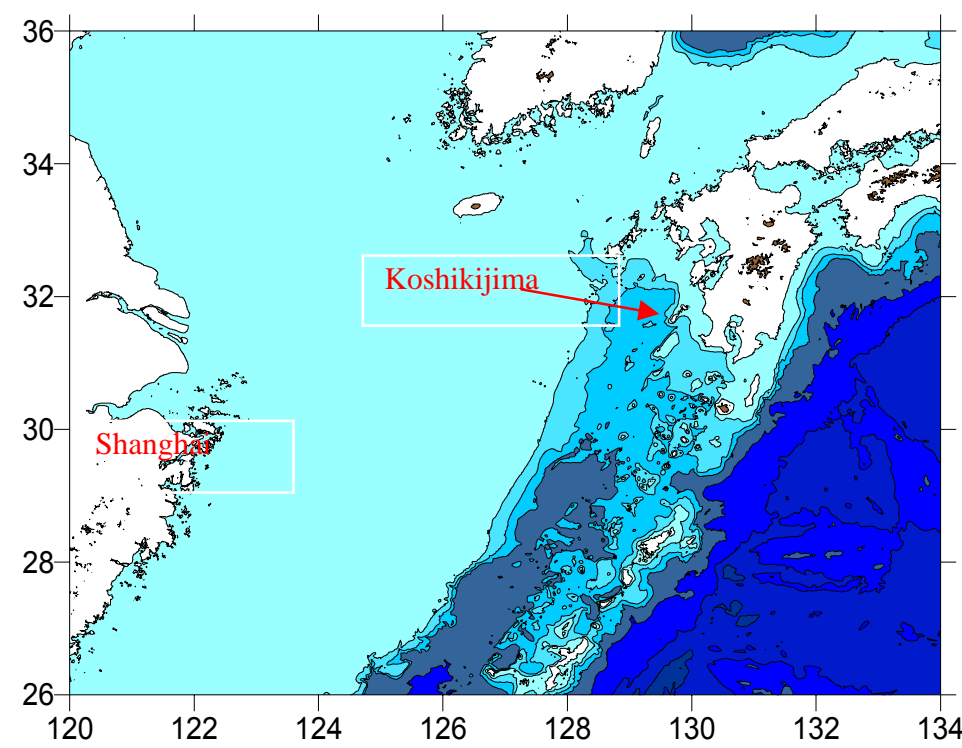

Figure 1 Loacation of Kami-Koshiki Island

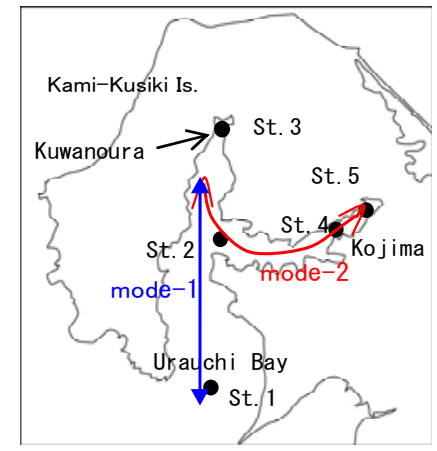

Figure 2 Study area and schematic illustration of seiche mode 

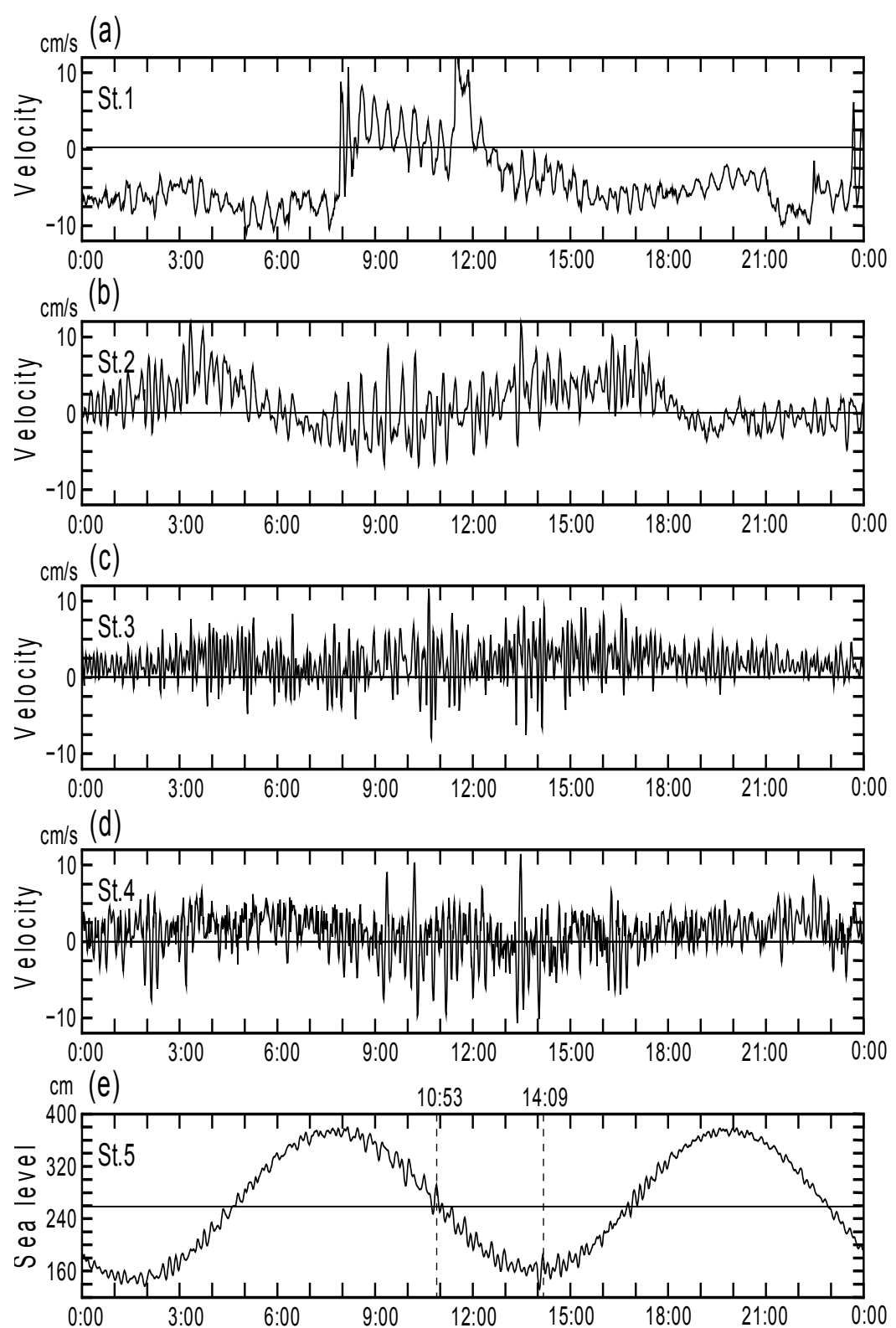

Oct. 14

Figure 3 Measured velocity data (main axis component) at St.1 St.4 and water surface fluctuation at St.5 


\section{Data Analysis}

At first, the tidal components were extracted using numerical low cut filter. FFT spectrum analyses were conducted for the temporal data of the axis velocities for St. $1 \sim$ St. 4 and the surface elevations at St. 5. The power spectra excluding tidal components are illustrated in Figure 4. The velocity power spectrum at the bay mouth St.1 has its peak at the period of 24.9min (Fig.4(a)). Meanwhile, the velocity spectra at the branching point St.2 and the inward parts St.3 and 4 have another peaks around the period of $7.2 \sim 12.6$ min of which magnitudes are around the same as that of the period of $24.9 \mathrm{~min}$ (Figs.4 (b) $\sim(\mathrm{d})$ ). The spectrum for water level fluctuation measured at Kojima fishing port (St. 5) also possesses around the same peaks as that of St.4 (Fig.4 (e)). It is also found in Figs. 4 that the peak energy for the period around 24.9 min keep almost same magnitude throughout the measuring areas from the mouth to the inward bays, however, the magnitudes for the periods of $7.2 \sim 12.6$ min increase as the measuring points go to the inward. Thus, the water level oscillation in Urauchi bay can be characterized as the composition of the two kinds of movement ; one is 24.9 minutes' oscillation which occurs throughout the T-type bay system, the other is 7.2 12.6 minutes' oscillation which occurs between both inward parts of the bay through the branching point. Hereinafter, the former oscillation of 24.9 min period is termed as mode- 1 oscillation, and the latter oscillation of $7.2 \sim 12.6 \mathrm{~min}$ is termed as mode- 2 oscillation.
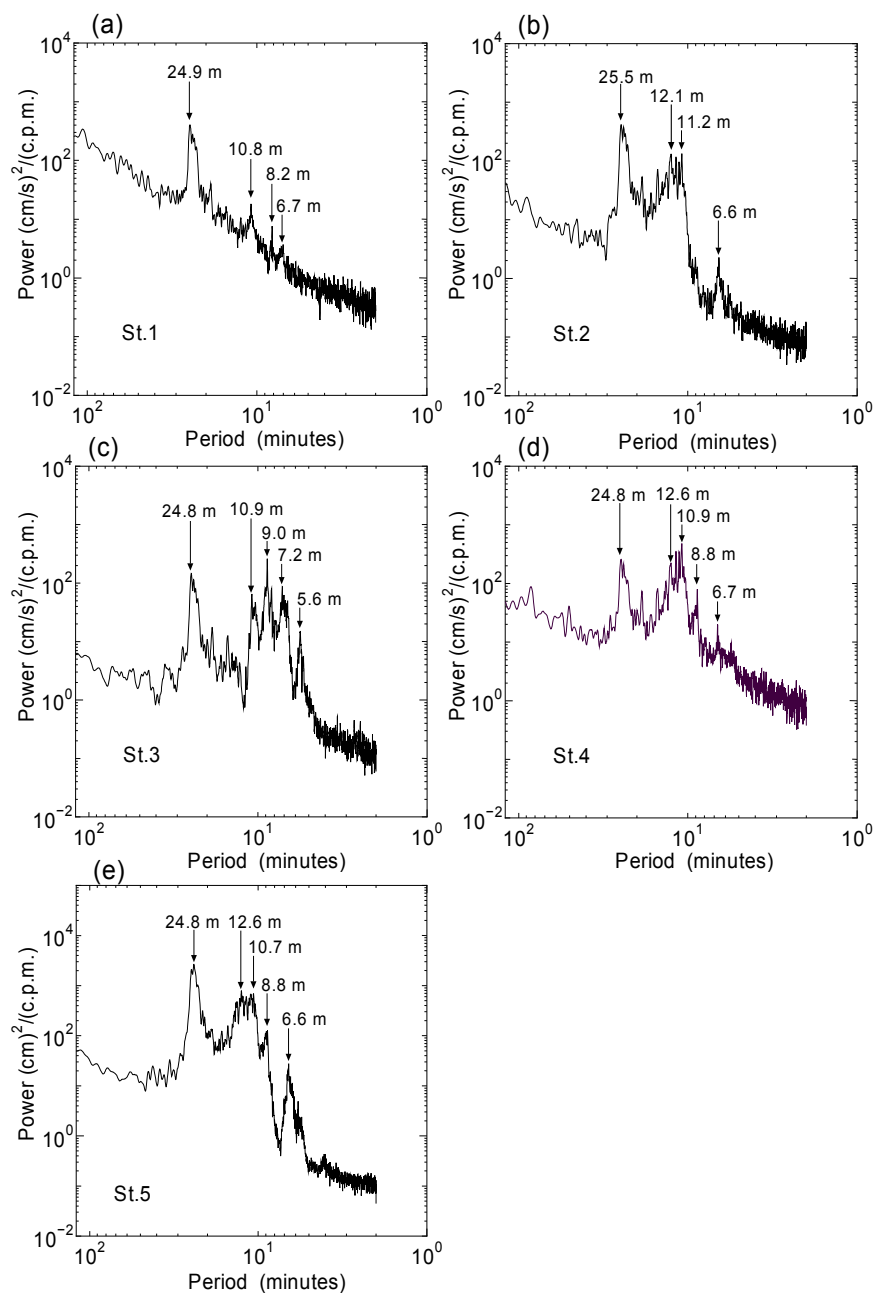

Figure 4 Power spectra of velocities (main axis component) at St.1 St.4 and water surface fluctuation at St.5 
Figure 5 shows the time histories of the velocity components taken on $9^{\text {th }}$ Oct., 2008 when secondary oscillations of $24.8 \sim 25.5$ min period were prevailed, whereas Figure 6 illustrates the time histories taken on $14^{\text {th }}$ Oct. when secondary oscillations of $7.2 \sim 12.6$ min were prevailed. It is found in Fig. 5 that the northward velocities in St.1 and St.3 are associated with eastward velocities in St. 2 and St.4 (which is shown by dotted lines in Fig.5), and the southward velocities in St.1 and St.3 couple with westward velocities in St.2 and St.4. That is because mode-1 is such an oscillation that possesses its node at the bay mouth and its anti-node at the inward ends. On the other hand, mode-2 oscillations are found in Fig. 6, in which the oscillations of $7.2 \sim 12.6 \mathrm{~min}$ become predominant as the point moves from the branching point to the inward. There are close correlations that northward velocities at St. 3 tend to be associated with eastward velocities at St.2 and St.4. Therefore, mode-2 can be characterized as an out-phase oscillation at the both ends of Kuwano-ura inlet and Kojima inlet. The oscillation resembles that occurs in a rectangular lake, where an anti-node appears at each end with a node at the central.

As shown in Figs.5 and 6, the secondary oscillations in Urauchi-bay vary their characteristics from day to day. To examine such time varying phenomena, wavelet spectrum analysis is effective. After extracting longer fluctuating components than 60min from the measured data, the wavelet spectrum analysis was conducted. Figure 7 illustrates the wavelet spectrum for the sea surface fluctuations at St. 5. In this figure, the red painted areas indicate great intensity of the fluctuation and blue areas show weak intensity. It is found in Fig. 7 that the great intensities of mode-1 oscillations

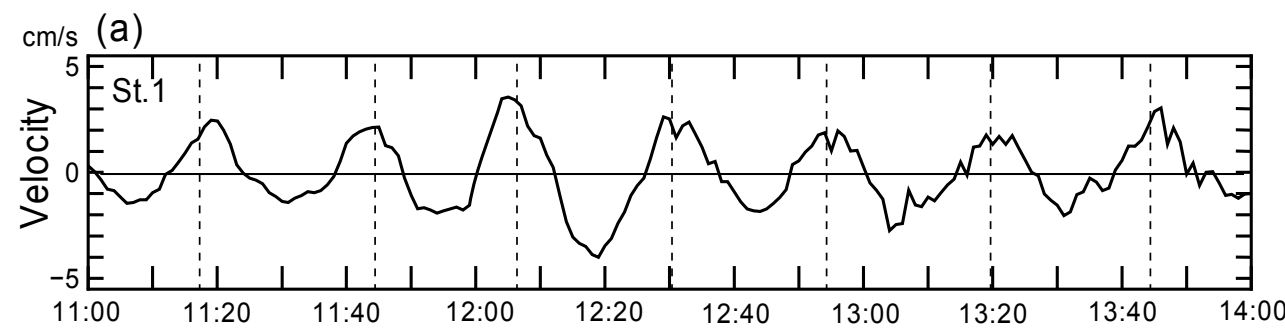

$\mathrm{cm} / \mathrm{s}(\mathrm{b})$

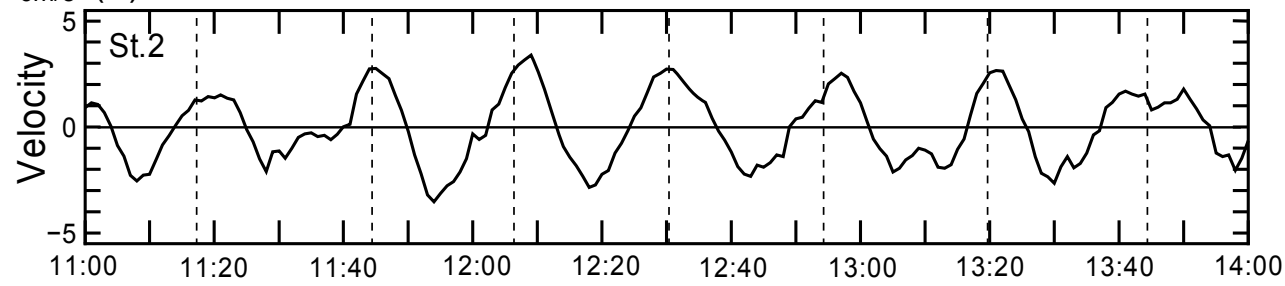

$\mathrm{cm} / \mathrm{s}(\mathrm{C})$

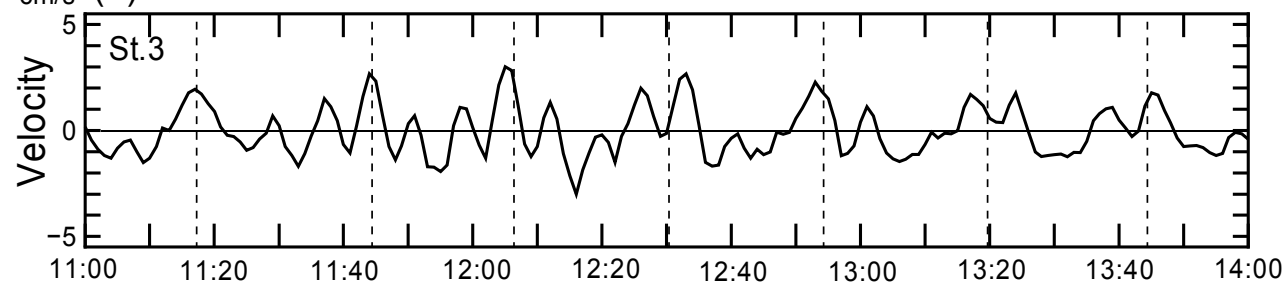

$\mathrm{cm} / \mathrm{s}(\mathrm{d})$

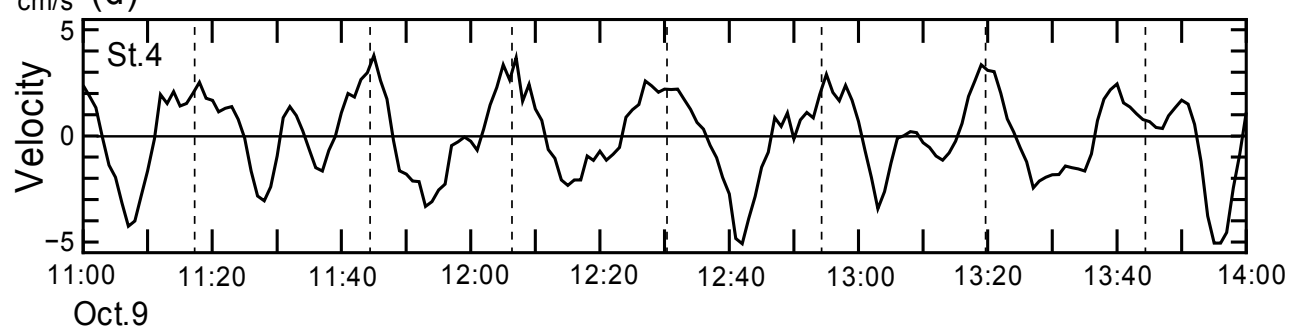

Figure 5 Time histories of velocity measured on 9 Oct., 2008 
(24.8 25.5 min period) and mode- 2 oscillations (7.2 12.6 min period) frequently appear and sometimes alternatively, especially in the durations $11 \sim 12,14$ and 17 Oct.

The time histories of the velocity fluctuations at St.1 $\sim$ St.4 for each component of $7 \sim 17 \mathrm{~min}$ and 17 30min are shown in Fig.8 and Fig. 9, respectively. The intensities of 17 30min oscillations obtained on $7^{\text {th }}$ Oct. do not vary so much among the four stations (Fig.8), meanwhile, the corresponding data of $7 \sim 17$ min oscillations (Fig.9) changes their magnitudes considerably; the minimum was obtained at St.1, then increased as the stations move inward (from St.2 towards St.4). The magnitude at St.4 became around 5 times as much as that at St.1. Comparison on the magnitudes of the St.1 velocity between Fig.8 and Fig.9 (note that the ordinates have different scale) indicates that the intensity of $7 \sim 17$ min component becomes smaller than that of $17 \sim 30 \mathrm{~min}$ component. Meanwhile, the magnitude of the St.4 velocity shows that the intensity of $7 \sim 17$ min component overtakes that of $17 \sim 30 \mathrm{~min}$ component considerably. The similar properties are found for the results taken on $11 \sim 12,14,17$ Oct.
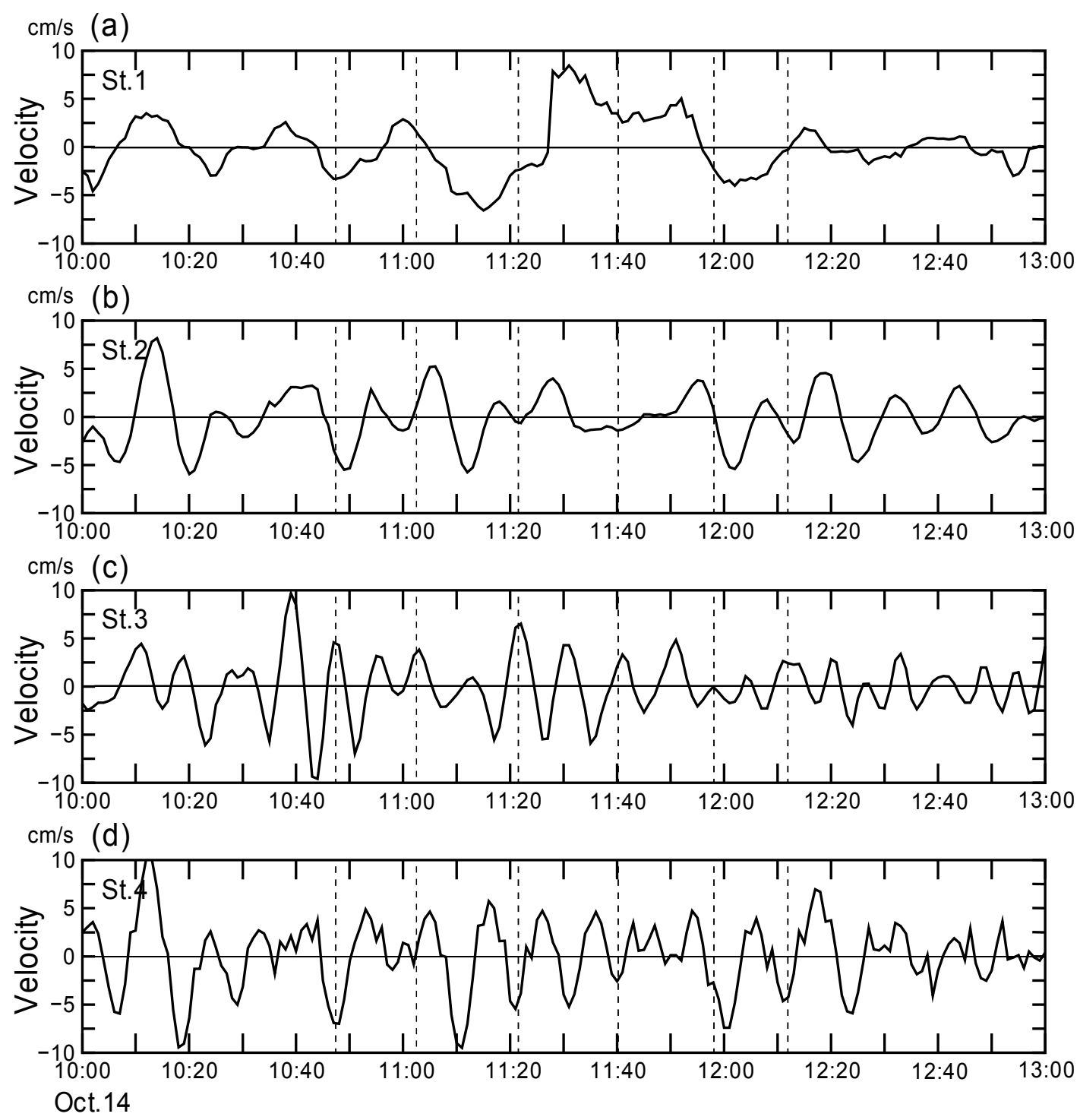

Figure 6 Time histories of velocity measured on 14 Oct., 2008 


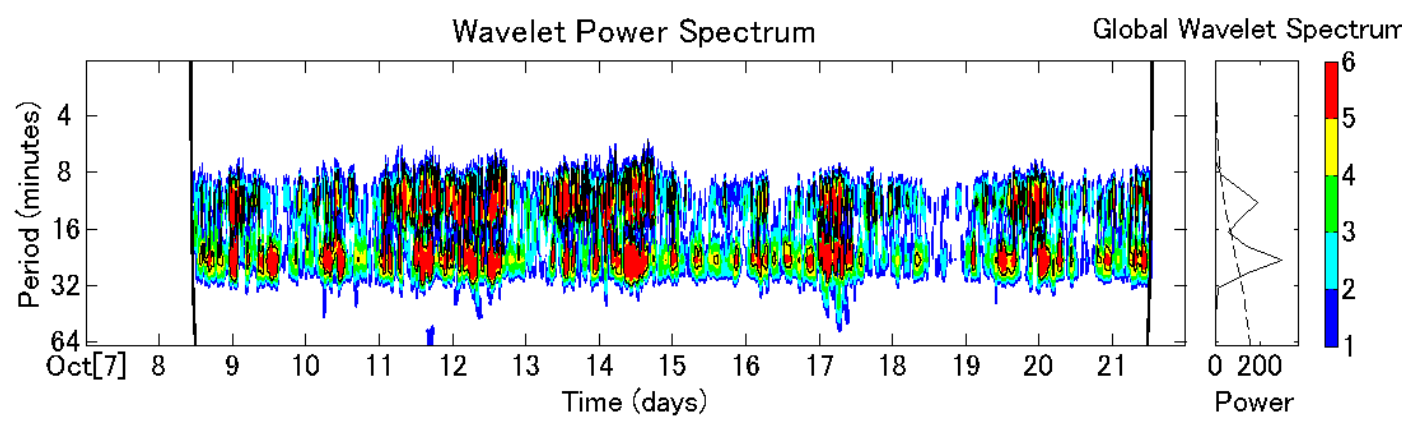

Figure 7 Wavelet spectrum of water surface fluctuation measured at St.5
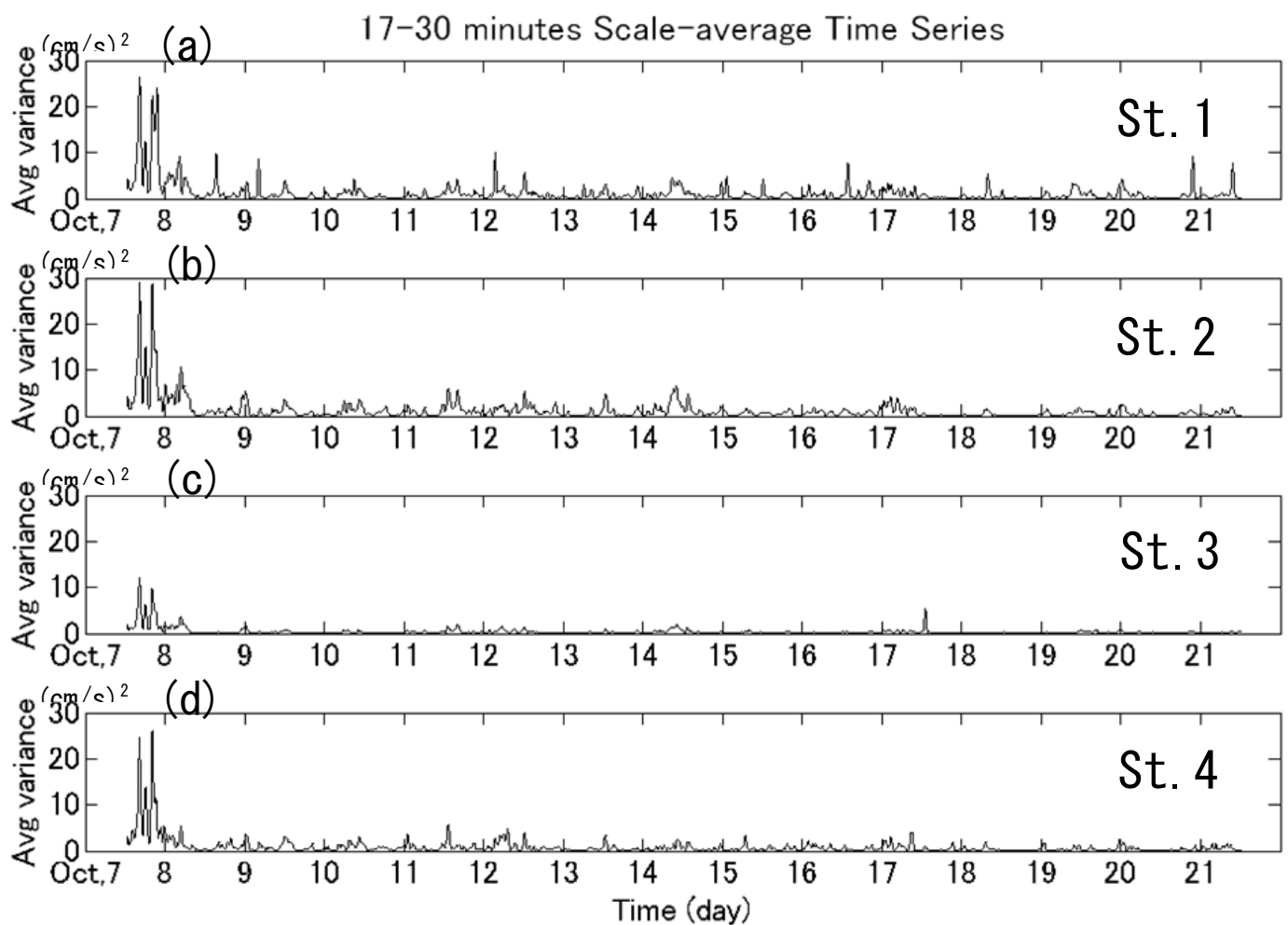

Figure 8 Time histories of velocity fluctuating intensity for $17 \sim 30$ minutes component

After all, for a T-shape bay such as Urauchi bay, there are two dominant modes in the oscillations; mode-1 oscillates having its node at the bay mouth and anti-node at the inward parts, and mode-2 oscillates between two inner inlets with its node at the branching point. Figures 8 and 9 indicate that the amplification rate of mode- 2 oscillation becomes greater than that of mode- 1 , thus, in the inward parts of the bay, mode-2 prevails over mode- 1 . However, as found in Fig. 4 (b) (e), the spectra in the periodic region 7.2 12.6 min might be composed of multiple peaks such as $12 \mathrm{~min}$, $11 \mathrm{~min}$ and $9 \mathrm{~min}$. This result might be considered that there exist the second or higher mode oscillation modes besides the above mentioned two fundamental eigen modes. Since the higher mode oscillations, however, were not clearly identified in the present measured data, only the mode- 1 and mode- 2 oscillations are discussed in the following. 

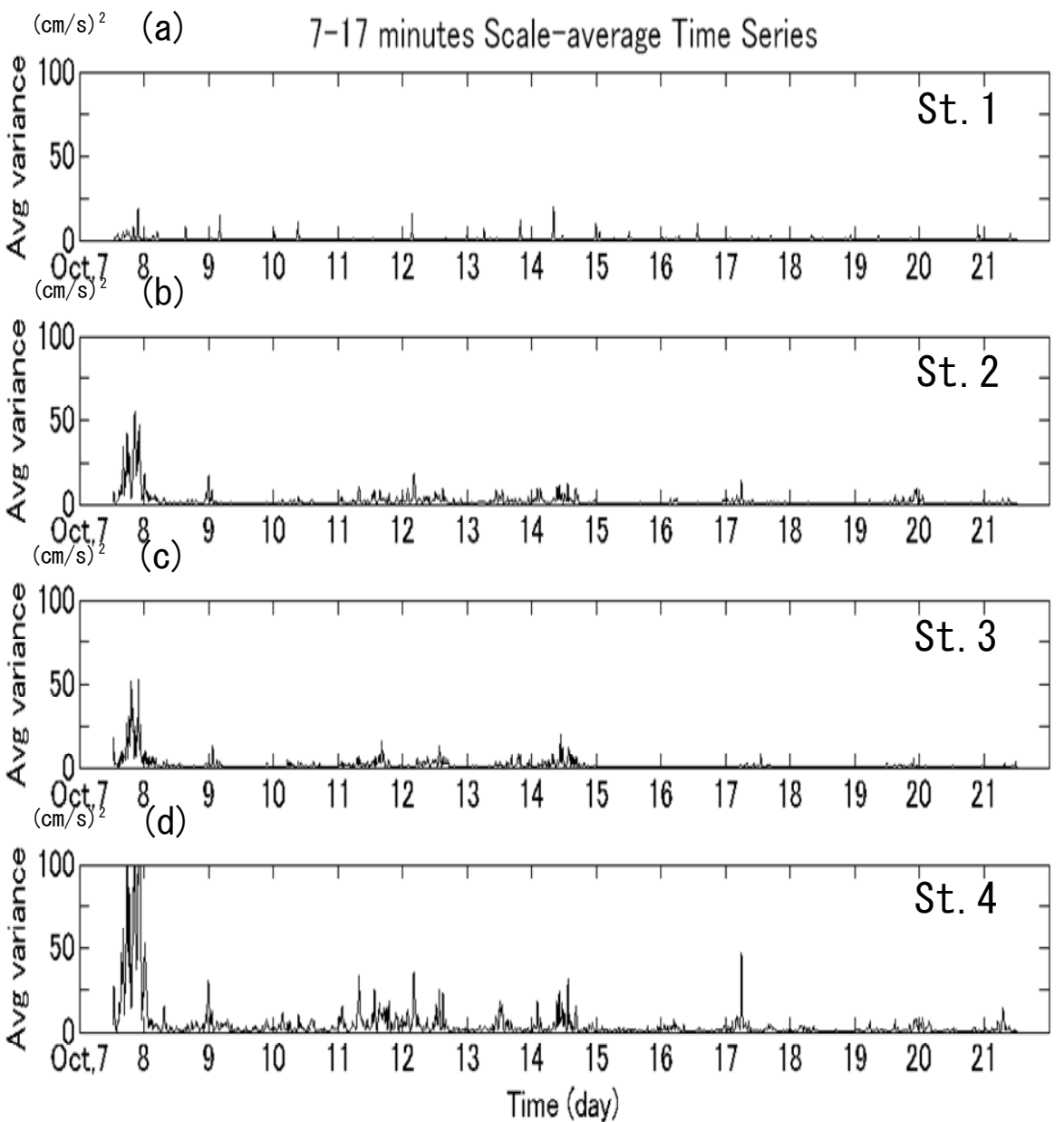

Figure 9 Time histories of velocity fluctuating intensity for $7 \sim 17$ minutes component

\section{NUMERICAL ANALYSIS}

In order to confirm the unique oscillating characteristics inherent to the T-shape bay obtained by the field observations, numerical analyses were conducted using the real bathemetry of the study area. The basic equations are non-linear shallow water equations as follows:

$$
\begin{aligned}
& \frac{\partial \eta}{\partial t}+\frac{\partial}{\partial x}[(\eta+h) U]+\frac{\partial}{\partial y}[(\eta+h) V]=0 \\
& \frac{\partial U}{\partial t}+\frac{\partial U^{2}}{\partial x}+\frac{\partial U V}{\partial y}=f V-g \frac{\partial \eta}{\partial x}+A_{h}\left(\frac{\partial^{2} U}{\partial x^{2}}+\frac{\partial^{2} U}{\partial y^{2}}\right)-\frac{K U \sqrt{U^{2}+V^{2}}}{\eta+h} \\
& \frac{\partial V}{\partial t}+\frac{\partial U V}{\partial x}+\frac{\partial V^{2}}{\partial y}=-f U-g \frac{\partial \eta}{\partial y}+A_{h}\left(\frac{\partial^{2} V}{\partial x^{2}}+\frac{\partial^{2} V}{\partial y^{2}}\right)-\frac{K V \sqrt{U^{2}+V^{2}}}{\eta+h}
\end{aligned}
$$

in which, $U, V$ is $\mathrm{x}$ - and y- directional depth averaged velocity, respectively, $\eta$ is the water surface elevation, $h$ is the still water depth, $f$ is the Coriolis' parameter, $g$ is the gravitational acceleration, $A_{h}$ is the horizontal diffusion coefficient and $K$ is the bottom friction coefficient. In the present calculations, $A_{h}$ is set as $20 \mathrm{~m}^{2} / \mathrm{s}, K$ is 0.0026 and the Coriolis' force is herein ignored.

The calculation domain is almost same as that shown in Fig. 2, of which extension is 
$8.1 \mathrm{~km} \times 7.35 \mathrm{~km}$. The grid size is set as $\Delta x=\Delta y=50 \mathrm{~m}$. The incident waves are given at the left side boundary of the domain, and the radiation conditions to ensure non-reflection are given at the upper and lower boundaries as well as the left side boundary. The following two incident waves , which are designed to reproduce mode-2 and mode-1 oscillation in Urauchi bay respectively, are given from the left side boundary.

(1) wave period $T=12 \mathrm{~min}$, amplitude $a_{0}=20 \mathrm{~cm}$

(2) wave period $T=27 \mathrm{~min}$, amplitude $a_{0}=20 \mathrm{~cm}$

For the above two cases, the calculated water levels and velocity vectors are illustrated in Fig. 10 and Fig.11. Figure 10, for the case of $T=12 \mathrm{~min}$, shows that the water level at the one inner inlet rises up whereas the water level at the other inlet goes down. It is also noticed that the water level at the branching point keeps almost the still water level, which indicates forming a nodal point. This results are considered to reproduce the mode- 2 oscillation that the water levels at both ends behave a see-saw type oscillation. Figure 11, for the case of $T=27 \mathrm{~min}$, illustrates that the water levels at both inner inlets rise up and fall down simultaneously. At the entrance of the bay, the water level does not fluctuate so much, which shows that a node is forming there. The characteristics agree with that mode- 1 oscillation where unti-nodes are forming at both inlet ends, and the whole water mass in the T-shape bay fluctuates as a fundamental eigen mode.

The authors' group conducted the similar numerical calculations as the present study for the same study area by varying the incident wave period (Shirahashi et al., 2008). The results can be summarized in terms of an amplification factor $K$, which is defined by a ratio of the oscillation height at the focused point $H$ against the incident oscillation height at the bay entrance $H_{i}$. Figure 12 shows the amplification factors at both inlet-ends of the present study; Kojima point and Kuwanoura point. The abscissa is the period of the incident waves. It is found in the figure that the curves of the amplification factor have twin peaks; the first peak around 1600s ( 27min) corresponds to mode-1, the second peak 700s ( 12min) corresponds to mode-2. Comparing Fig.12 and Fig. 4 (a) and (e) , the spectra measured at the entrance and Kojima point, both numerical and field results agree well with regards to the peak frequencies and possessing multiple peaks. However, the numerical results show that the amplification factor at the peak 1600s becomes greater than that of 700s. This implies that the mode- 1 oscillation is dominant over mode- 2 in the numerical results. Whereas, the measured results suggest that the amplification of the wave energy in mode- 2 could exceed that in mode- 1 . The difference might be resulted from the assumptions of the numerical analysis where the incident waves were given as continuous sinusoidal waves with a single period component. In the field, however, composite and transient oscillations are arriving to the study domain. Furthermore, the present numerical analysis needs some improvements to reproduce the complicate land topography and man-made structures like jetties by refining the grid size. 

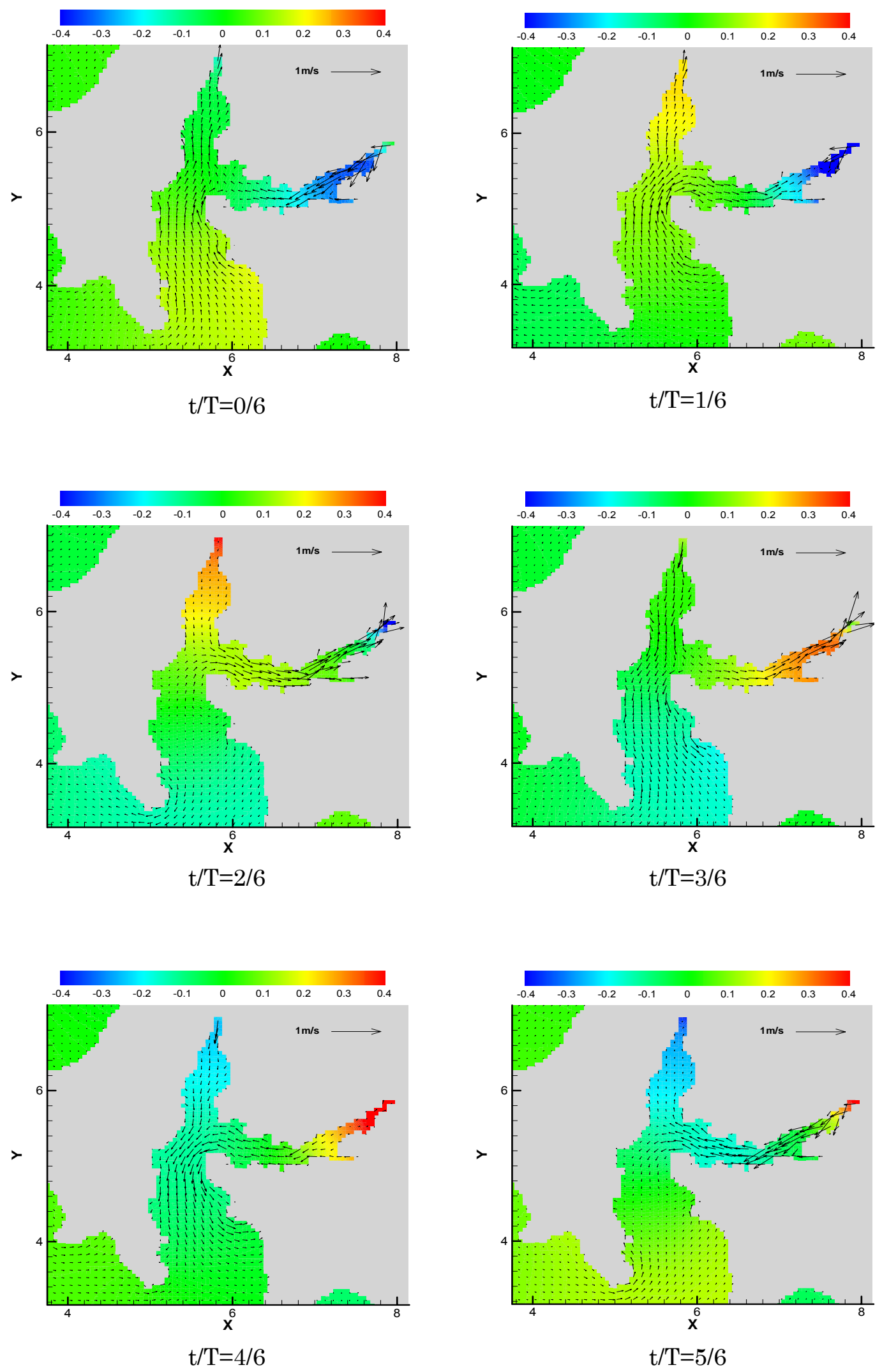

Figure 10 Spatial distributions of water levels and velocity vectors ( $\mathrm{T}=12 \mathrm{~min})$ 

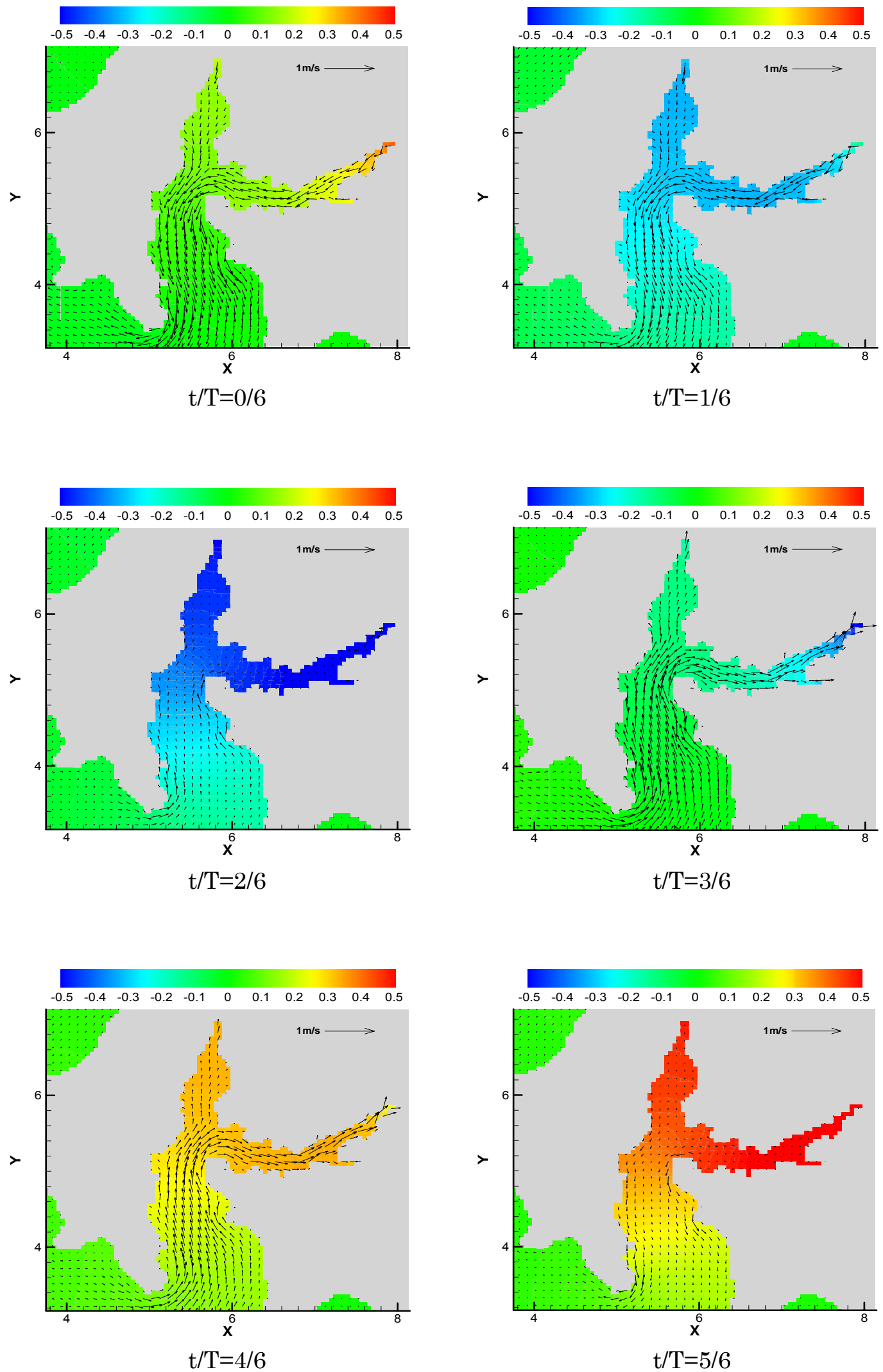

Figure 11 Spatial distributions of water levels and velocity vectors ( $\mathrm{T}=27 \mathrm{~min}$ ) 


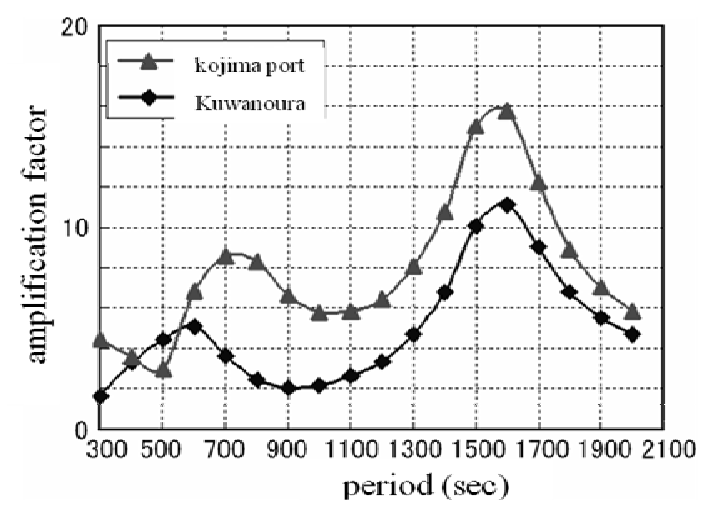

Figure 12 Amplification factors at both inner inlets

\section{CONCLUSIONS}

Field observations have revealed unique oscillating characteristics in a T-shape bay. There exist two predominant modes of seiche motions; mode-1 oscillates having its node at the bay mouth and anti-node at the inward parts, and mode-2 oscillates between two inner inlets with its node at the branching point.

Numerical analysis provides basically consistent results with the observed results. Mode-1 and mode-2 oscillations can be reproduced by the numerical simulation. Both peak periods are well reproduced quantitatively. In terms of the amplification factor, the numerical results showed that mode- 1 oscillation is predominant over mode- 2 oscillation, whereas, the observed results sometimes show the opposite predominance between the two modes.

These results can be utilized in future as the mitigation activities towards the destructive seiche events by developing the forecasting system, finding the appropriate positions for mooring ships or setting fishing cages. For this purpose, the following subjects should be done. The first, the generation mechanism of the seiche events needs to be clarified from both aspects from atmospheric and ocean dynamics. Second, the resonant mechanism of the seiche also requires to be investigated by adopting more systematic field observation system. Finally, the numerical analysis needs further refinements to include the fine topography and effects of the presence of jetties and large fishing cages. The interaction between atmospheric disturbance and ocean surface fluctuations should be considered. The present study is ongoing to investigate above mentioned subjects, the authors wish to present the further developments in future occasions.

\section{REFERENCES}

de Jong, M. P. C. and J. A. Battjes (2004): Low frequency sea waves generated by atmospheric convection cells, J. Geophysical Res., Vol. 109, C01011, pp.1-18.

Hibiya, T. and K. Kajiura (1982) : Origin of the Abiki phenomenon (a kind of seiches) in Nagasaki bay, J. Oceanographical Society of Japan, Vol. 38, pp.172-182.

Shirahashi, T., T. Kakinuma, T. Asano and M. Sato (2008): Numerical simulation of long-period oscillation in a branching bay of Koshiki islands, Annual Journal of Coastal Engineering, JSCE, pp.216-220 (in Japanese).

Vilibic, I., S. Monserrat, A. Rabinovich and H. Mihonovic (2008) : Numerical modeling of the destructive meteotsunami of 15 June, 2006 on the coast of the Balearic Islands, Pure and Applied geophysics, Vol.165, pp.2169-2195. 